\title{
Competencias profesionales y organizaciones multiculturales: identificación e instrumentos de medida de la competencia intercultural
}

\author{
Ana Carballal \\ Universidad Nebrija \\ acarball@nebrija.es \\ M. José Pinillos \\ Universidad Rey Juan Carlos \\ mariajose.pinillos@urjc.es
}

Resumen: Este trabajo analiza el concepto de competencia intercultural entendida como una competencia profesional para trabajar de manera efectiva en entornos multiculturales, cada vez más comunes en el mundo globalizado. Se trata de una revisión de las distintas maneras de identificación y evaluación de la competencia intercultural, así como de los diversos modelos e instrumentos de medida que sirven para evaluar la competencia intercultural. A través de este estudio se muestra que, a pesar de la importancia de estas habilidades, existen dificultades por parte de las organizaciones a la hora identificar y formar este tipo de competencias. Esto se debe principalmente a dos razones. Por una parte, una minimización de las diferencias culturales que conduce a la falsa creencia de que debido a la globalización estamos abocados a una homogeneización cultural. Por otra parte, la falta de un denominador común a la bora definir la competencia intercultural y de entender su aplicación en entornos profesionales, que, a su vez, provoca un desconocimiento de los beneficios profesionales que esta competencia supone para el éxito empresarial.

Palabras clave: diversidad cultural, competencia intercultural, globalización, desarrollo de babilidades profesionales, instrumentos de medida, organizaciones multiculturales. 
Professional competences and multicultural organizations measurement instruments of intercultural competence

\begin{abstract}
This paper analyzes the concept of intercultural competence understood as a professional skill needed to be able to work effectively in the multicultural environments that are increasingly common in the globalized world. It reviews the different ways of identifying and evaluating intercultural competence, as well as the various models and measuring instruments that are used to assess it. This study shows that, despite the importance of these skills, organizations experience difficulties in identifying and training people in these types of skill. This is mainly due to two reasons; on the one hand, the minimization of cultural differences leads to the false belief that globalization inevitably result in cultural homogenization, and on the other hand, there is a lack of a common denominator for defining intercultural competence and understanding its application in professional environments that, in turn, leads to ignorance of the benefits that this skill brings in terms of professional and business success.
\end{abstract}

Keywords: cultural diversity; intercultural competence; globalization; professional skills development; measurement instruments; multicultural organizations. 


\section{Introducción}

La competencia intercultural se ha convertido en una capacidad imprescindible para el entendimiento de personas de distintas culturas, tanto en el ámbito personal como profesional. El Consejo de Europa presenta la competencia intercultural como una habilidad esencial que debe ser desarrollada por todos los individuos para posibilitar el diálogo intercultural. Barrett et alii (2013) reconocen que el entendimiento mutuo y la interculturalidad son más importantes que nunca, ya que los prejuicios, la discriminación y los discursos de odio se han vuelto habituales en las sociedades actuales y los partidos que defienden ideas extremas parecen estar en auge.

En lo que se refiere a las empresas y organizaciones es destacable que, en su mayoría, se encuentran inmersas en un proceso globalizador que se viene acrecentando ya desde hace décadas. Por ello la búsqueda de estrategias para favorecer el intercambio comercial que acompaña a la globalización pasa indudablemente por el desarrollo de los recursos humanos a distintos niveles. Dentro de este campo, la gestión de la diversidad cultural se presenta como uno de los grandes retos del siglo xxi. Todo apunta a que el día de mañana la gran mayoría de los entornos laborales serán de índole multicultural y, bien sea dentro o fuera de las fronteras nacionales, los profesionales del futuro se verán envueltos en relaciones comerciales y laborales interculturales donde tendrán que maniobrar con destreza. Es muy posible que solo aquellos que estén preparados para afrontar el reto que supone el entendimiento entre culturas serán capaces de desarrollar con éxito y sostenibilidad el futuro de sus organizaciones.

Así pues, existe una incipiente necesidad de estudiar en profundidad las competencias profesionales necesarias para optimizar el rendimiento profesional en entornos internacionales o multiculturales. Por ello es fundamental analizar la competencia intercultural, dentro del marco de las competencias profesionales, ya que todavía existen pocas investigaciones orientadas a este respecto. A pesar de que se trata de una competencia imprescindible en el mundo global, el marco conceptual es todavía impreciso. Las definiciones de competencia intercultural son múltiples y variadas y no parece existir un consenso ni a nivel académico ni profesional sobre cómo identificarla, evaluarla o desarrollarla. Asimismo, es destacable que los estudios empíricos sobre la competencia intercultural hasta el momento se limitan mayoritariamente a poblaciones de expatriados o estudiantes internacionales, aunque se reconoce cada vez más la necesidad de ampliar el espectro a cualquier persona que viva y trabaje en un entorno culturalmente heterogéneo. En este trabajo pretendemos contribuir a reducir este vacío y para ello analizamos la competencia intercultural, entendida como una habilidad pro- 
fesional. También estudiamos su identificación y medición con el fin de aportar mayor conocimiento en lo que respecta a la selección de talento intercultural y la gestión de la diversidad cultural en las organizaciones.

A tal efecto este trabajo se ha estructurado como sigue. En primer lugar, presentamos una revisión de la literatura. Seguidamente se define el concepto de competencia intercultural y, en el siguiente apartado, se trata la relación entre competencia intercultural y el éxito profesional en entornos multiculturales. Más adelante, se describen los modelos de competencia intercultural para analizar, en el apartado siguiente, las herramientas para identificar y evaluar la competencia intercultural.

\section{Revisión de la literatura}

El estudio de la competencia intercultural a nivel profesional es reciente y su evaluación y desarrollo parecen encontrarse todavía en una etapa de desarrollo inicial. Las teorías que explican las diferencias culturales basadas en investigaciones empíricas sólidas y que identifican patrones culturales en las distintas sociedades apenas tienen unas décadas. Algunos especialistas, como el psicólogo social holandés Geert Hofstede, pionero en este campo a principios de los años ochenta del siglo pasado, han permitido a través de sus múltiples y extensas investigaciones acrecentar el interés por la interculturalidad aplicada a ámbitos profesionales y ampliar el número de especialistas e investigadores en distintas partes del mundo. Es, sin embargo, destacable que el concepto de competencia intercultural, entendida como una habilidad profesional de índole general, es relativamente nuevo.

En cuanto a su desarrollo histórico es importante señalar que la competencia intercultural adquiere mayor importancia en los años noventa del siglo pasado debido al rápido crecimiento de la internacionalización empresarial y la globalización. Empiezan a aparecer entonces diferentes definiciones y también modelos de competencia internacional. En los últimos años, los estudios sobre competencias globales y habilidades interculturales se han multiplicado, y, con ellos, su definición.

Sobre las publicaciones específicas que hacen referencia a las habilidades interpersonales necesarias para trabajar con éxito en un ámbito intercultural, encontramos que muchas giran en torno a cómo tratar de identificarlas y de formarlas para alcanzar un buen rendimiento profesional en un entorno internacional. Destaca la necesidad de un consenso sobre cuáles son exactamente estas habilidades y características deseables en un profesional que trabaje en un ámbito intercultural dentro o fuera de sus propias fronteras. Una mayoría de publica- 
ciones alude de forma específica a trabajadores expatriados y a su éxito o fracaso en misiones internacionales. Dado que el índice de fracasos en este ámbito es alto, los diferentes autores, ya en las últimas décadas del siglo $\mathrm{xx}$, coincidían en que es esencial comprender las razones que convierten a una persona en idónea para poder desempeñar un trabajo en un entorno internacional. Kealey y Ruben (1983), por ejemplo, tratan la selección de personal para entornos internacionales y muestran su preocupación por la gran inversión en tiempo y dinero empleada para preparar a los trabajadores expatriados y a sus familias. Los autores destacan las consecuencias que un fracaso o un regreso anticipado pueden suponer para la organización y para el trabajador. Tung (1981) estudia también la adaptación, el rendimiento de trabajo y el entrenamiento para entornos interculturales, defendiendo que hay diferentes criterios para la selección de personal dependiendo del grado de contacto con la nueva cultura y la duración de la misión internacional. Hawes y Kealey (1981) analizan al personal expatriado en países en desarrollo. Sus resultados muestran que la eficacia intercultural está muy relacionada con las habilidades interpersonales, pero también con el sentido de la identidad y las expectativas previas que un individuo tiene antes de su partida.

Hannigan (1990) realiza una revisión de la literatura y sintetiza lo que se ha publicado acerca de las habilidades, actitudes y rasgos que se relacionan con la eficacia intercultural. En su estudio amplía los elementos anteriores con algunos nuevos: capacidad de comunicarse, capacidad para establecer y mantener relaciones, orientación al conocimiento, visión del mundo, empatía cultural, capacidad lingüística, flexibilidad y una visión realista de la cultura de destino y su gestión de la organización. También alude a los factores que tienen una correlación negativa, como la ansiedad, el autoritarismo, el perfeccionismo, la rigidez, el etnocentrismo, la estrechez de miras y los comportamientos egocéntricos.

Uno de los estudios más destacables es el de Arthur y Bennett (1995), donde los autores tratan de averiguar qué factores son claves para conseguir el éxito profesional de un expatriado en una misión internacional. Parten de la hipótesis (bastante extendida a día de hoy) de que los factores psicosociales son más importantes en cuanto al rendimiento del trabajo en un entorno internacional que los propiamente técnicos o relacionados con el conocimiento específico. Siguiendo la línea de otros autores, proponen prestar más atención a la selección de personal y al entrenamiento intercultural. En lo que se refiere al entrenamiento, Arthur y Bennett (1995) plantean que la formación intercultural no está tan extendida como podría pensarse, entre otras cosas, porque los gestores consideran que el entrenamiento no es efectivo. La razón por la que se piensa de esta manera es, precisamente, porque no existe una idea clara de qué y cómo se debe entrenar. En otras palabras, no tienen claro qué factores se deben tener en cuenta 
para alcanzar el éxito en entornos internacionales y/o interculturales y cuáles son las competencias específicas que convendría desarrollar. Para Saso et alii (2017), cómo abordar el aprendizaje de la competencia intercultural es la cuestión más compleja.

Shaffer et alii (2006) hacen hincapié en este problema y señalan que, a pesar de la importancia que tiene la selección de expatriados para el éxito de la experiencia, muchas empresas todavía basan su selección tan solo en los conocimientos técnicos y en la disposición del empleado (o su familia) de expatriarse. Los autores insisten en que los costes y las pérdidas en caso de fracaso son tan altos que ya no se debería seleccionar a este tipo de personal basándose simplemente en métodos heurísticos.

Algunos autores destacan la competencia intercultural como una de las competencias profesionales necesarias para mejorar la empleabilidad de los estudiantes (Busch, 2009), pero, además, afirman que se trata de una competencia muy demandada en cualquier organización internacional o nacional en proceso de internacionalización. Como explica Espinosa (2009: 108): «Las competencias interculturales son un conocimiento estratégico para los que deban trabajar en entornos internacionales, conocer la cultura de aquellos con los que va a interactuar puede facilitar desde negociaciones trascendentales para la compañía hasta hacer más confortable su día a día».

Irving (2010) lleva esta competencia más allá de los límites de la internacionalización y explica que, si bien la competencia intercultural se solía considerar como algo necesario solo para las personas que trabajaban en ámbitos internacionales, en la actualidad nos encontramos con la necesidad de dotar a la mayoría de los dirigentes y personal de una organización con competencia intercultural. Para Bakalis y Joiner (2004), las organizaciones de hoy en día necesitan personas abiertas a la diversidad, que puedan prosperar en situaciones inciertas y complejas y que se adapten a los cambios.

No obstante, la influencia del factor cultural en las organizaciones está poco estudiada y requiere de una mayor atención por parte de los investigadores, profesores, gestores y, en especial, de los profesionales de recursos humanos. Llamazares García-Lomas (2011) hace referencia a los estudios relativos a la comunicación intercultural como una vía para promover el diálogo entre culturas, pero indica que en español son todavía pocos los autores y las publicaciones que analizan la influencia de la cultura en la forma de hacer negocios.

No cabe duda, por tanto, que para muchos autores resulta imprescindible reclutar y formar a profesionales capaces de moverse fácilmente en el mundo global y ser eficaces en entornos internacionales o multiculturales. Sin embargo, la pregunta sigue girando en torno a cómo evaluar $y$, por consiguiente, desarrollar 
este tipo de habilidades. Si bien es verdad que la mayoría de los estudiosos mencionan conceptos y características hasta cierto punto similares, no parece existir un denominador común del cual partir para resolver esta problemática.

\section{El concepto de competencia intercultural: la necesidad de un denominador común}

La competencia intercultural es una competencia imprescindible para vivir y trabajar el mundo global, ya que la diversidad cultural es cada día más común en los ámbitos profesionales. No obstante, los conceptos y componentes relacionados con este tipo de habilidad son múltiples y muy variados, lo que dificulta su identificación y evaluación. Por tal razón, la necesidad de identificar o desarrollar este tipo de competencia no está tan clara como cabría esperar. En algunos casos, incluso se ha llegado a pensar que el mundo de los negocios se ha ido homogeneizando, pasando las diferencias culturales a un segundo plano. Como indica Fanjul (2010), esa estandarización de las pautas culturales es una hipótesis muy arriesgada, porque sobrevalora las similitudes entre culturas y su grado de aproximación e infravalora el apego de mucha gente por mantener sus pautas culturales diferenciales.

Para analizar mejor esta cuestión es imprescindible comenzar por definir qué entendemos por cultura. Un gran número de académicos de distintas ramas del conocimiento, como pueden ser la filosofía, la historia, la antropología, la comunicación o la psicología, entre otras, han tratado de definir el concepto cultura. Lo cierto es que no resulta sencillo encontrar una única definición en la que basarse, ya que no existe un consenso en la literatura al tratarse de un concepto amplio y con múltiples interpretaciones. Algunos autores definen la cultura como una forma de vida, aprendida y compartida por un grupo de personas, que está compuesta de patrones, valores, normas e incluso objetos materiales comunes (Liu, Volcic y Gallois, 2015).

El famoso antropólogo e investigador intercultural estadounidense Edward T. Hall se refiere a la cultura en su libro La dimensión oculta como «experiencias profundas, comunes e implícitas, que los miembros de una determinada cultura comparten, comunican sin saberlo, y forman el escenario desde el que son juzgados todos los demás acontecimientos» (Hall, 1982: IX). Para Geert Hofstede: «[... [ la cultura es la programación colectiva de la mente humana que distingue a los miembros de un grupo de los de otro». En un sentido amplio del término la cultura es un fenómeno colectivo que se comparte con personas que viven o han vivido en el mismo entorno social en el que han adquirido dicha cultura (Hofstede, Hofstede y Minkov, 2010)Geert Hofstede proposed four dimensions 
on which the differences among national cultures can be understood: Individualism, Power Distance, Uncertainty Avoidance and Masculinity. This volume comprises the first in-depth discussion of the masculinity dimension and how it can help us to understand differences among cultures. The book begins with a general explanation of the masculinity dimension, and discusses how it illuminates broad features of different cultures. The following parts apply the dimension more specifically to gender (and gender identity. Así pues, estamos programados por el grupo cultural al que pertenecemos para entender el mundo de una determinada manera.

La cultura es, por tanto, un conjunto de valores y creencias, en ocasiones invisibles, adquiridos y transmitidos por un grupo de personas que producen comportamientos y normas específicas y que resultan difíciles de entender para miembros de otros grupos. La cultura influye enormemente en nuestra manera de comprender el mundo, definiendo nuestro concepto del tiempo, del espacio, del medio ambiente o de las relaciones. Observamos a las personas que nos rodean e interpretamos sus creencias y comportamientos desde una perspectiva unilateral, es decir, desde nuestro propio contexto cultural.

Es importante distinguir esta definición de cultura de la que se utiliza habitualmente como sinónimo de civilización. Como señala Akli (2013), la civilización se puede estudiar a través de libros, películas, fotografías y otras fuentes primarias y secundarias $y$, aunque es muy interesante como punto de partida para desarrollar la competencia intercultural, no está supeditada a ella. Sin embargo, el conocimiento de la cultura, aunque también requiere de formación, es algo más experimental que pasa por una observación previa y una sensibilización cultural y de respeto al otro, cuestiones no estrictamente necesarias para el estudio de las civilizaciones.

En la actualidad, en ámbitos profesionales, incluso en los internacionales, se tiende a equiparar la competencia intercultural más con el conocimiento específico de determinadas culturas, es decir, con la civilización, que con el manejo efectivo de la diversidad cultural. Esta cuestión se aprecia, por ejemplo, en algunos libros convertidos en grandes éxitos, como es el caso del de Morrison y Conaway (2006), donde los autores presentan información detallada y organizada por países para que el lector tome nota de aquellos comportamientos específicos (relacionados con gestos, vestimenta, saludos, etc.) que deben ser tenidos en cuenta a la hora de hacer negocios en el extranjero. De esta manera, a día de hoy, las organizaciones todavía suelen hacer más hincapié en el conocimiento específico sobre un país y en aquellas conductas visibles (deseables o indeseables) que en profundizar en valores y creencias o en habilidades interpersonales, como pueden ser la flexibilidad, la empatía o la apertura mental. 
Por todo ello, y para impedir que la competencia intercultural se limite a un plano superficial, es fundamental tratar de encontrar un terreno común a la hora de definirla y analizarla dentro de los entornos laborales. La dificultad, una vez más, radica en que las definiciones relacionadas con el concepto de competencia o habilidad intercultural son múltiples y diversas y, aunque se basan en ideas y conceptos similares, pueden variar de autor a autor.

Para definir las competencias necesarias para la gestión y el funcionamiento en entornos internacionales o multiculturales se utilizan, además, una multitud de términos que, en su mayoría, provienen del inglés. Así pues, se habla de competencia intercultural (intercultural competence), pero también de mentalidad global (global mindset), sensibilidad intercultural (intercultural sensitivity), conciencia intercultural (intercultural awareness), inteligencia cultural (cultural intelligence) $y$ personalidad multicultural (multicultural personality), entre otros conceptos, a veces entremezclados y cuya definición tiende a ser confusa.

Hammer et alii, por ejemplo, se refieren a la sensibilidad intercultural como la habilidad para discriminar y experimentar diferencias culturales relevantes $y$ a la competencia intercultural como la capacidad para pensar $y$ actuar de formas interculturalmente apropiadas. De esta manera, entienden que «a mayor sensibilidad intercultural, mayor potencial para ejercitar la competencia intercultural» (Hammer et alii, 2003: 422).

Bücker y Poutsma (2010), por su parte, definen la competencia para funcionar de manera efectiva en un entorno internacional como una conjunción de varios factores: conocimiento, habilidades, capacidades, personalidad y comportamiento. De esta manera buscan identificar lo que algunos autores denominan KSAO: knowledge, skills, abilities and other personality characteristics (Matsumoto y Huang, 2013; Chiu et alii, 2013). Johnson, Lenartowicz y Apud (2006) señalan que aquellos con deficiencias en estos factores pueden no reconocer siquiera la necesidad de adaptar su comportamiento. Van der Zee y Van Oudenhoven (2013) consideran que son las características de personalidad, tanto innatas como adquiridas gracias al entorno, las que predicen la competencia intercultural. Además, esta competencia requiere ser sensible, reflexivo y activo en la interacción con gente de otras culturas. A una persona interculturalmente competente le suele gustar tener contacto con extranjeros, puede trabajar de manera eficiente con ellos y realizar tareas sin que le suponga un estrés añadido (Brislin, 1993, citado en Behrnd y Porzelt, 2012).

Para Barrett et alii (2013), la competencia intercultural es una combinación de actitudes, conocimientos, entendimientos y habilidades que le permiten a uno comprender y respetar a personas culturalmente diferentes, responder de manera adecuada, eficaz y respetuosa al interactuar y comunicarse con ellas. 
También el hecho de establecer relaciones positivas y constructivas y reconocer la identidad cultural propia a través de la relación con otras culturas. En cuanto al conocimiento y entendimiento, los autores destacan conocer los valores y creencias de otras culturas, comprender la heterogeneidad de los grupos culturales, saber de la influencia de la lengua, de las diferencias en la comunicación dependiendo de la cultura y ser consciente de que existen ideas preconcebidas, prejuicios y estereotipos. En referencia a las habilidades, la competencia intercultural incluye: la capacidad de cambiar y adaptar el pensamiento y el comportamiento a distintos contextos y entornos, tomar otras perspectivas en consideración; demostrar empatía al entender y responder a los sentimientos de otras personas o ser capaz de interpretar las creencias, valores y prácticas de otras culturas. También mencionan habilidades lingüísticas y sociolingüísticas, la capacidad para mediar, traducir o interpretar en situaciones interculturales, así como gestionar situaciones donde exista una falta de comunicación. Con respecto a las actitudes, los autores proponen incluir la valoración del pluralismo y la diversidad; el ser curioso y abierto con interés por aprender de otras culturas; la empatía hacia personas culturalmente distintas respetando sus afiliaciones e identidades; tolerancia ante lo incierto o ambiguo, así como la búsqueda de oportunidades para tener más contacto intercultural y perspectivas culturales distintas (Barrett et alii, 2013).

Según Johnson et alii (2006), también es necesario que estos componentes se implementen y se pongan en práctica a través de la acción durante los encuentros interculturales. Las personas a menudo profesan actitudes y adquieren conocimientos y habilidades que no logran poner en práctica. Por esta razón, para poder atribuirle a un individuo competencia intercultural, debe también aplicar actitudes interculturales, conocimiento, entendimiento y habilidades a través de las acciones.

Así pues, existe una enorme variedad de perspectivas a la hora de definir un tipo de habilidades o características específicas que un profesional debe tener para trabajar de manera efectiva entre culturas. Además, el problema se materializa y se agudiza al tratar de medir estas habilidades o características. Como explica Deardorff (2006) en un su estudio con expertos académicos y administradores, para medir la competencia intercultural es preciso estudiar y analizar qué se entiende por ella y buscar un terreno común que permita proceder a su evaluación. 


\section{La competencia intercultural y su relación con el éxito profesional en ámbitos internacionales}

Dada la complejidad del proceso de internacionalización de una organización es fundamental priorizar la gestión de recursos humanos para poder identificar y desarrollar el talento y las habilidades interculturales de todos los empleados involucrados, de una manera u otra, en dicho proceso. Es importante para ello que la investigación se aplique a la evaluación y al entrenamiento de habilidades interculturales en personas o grupos que tienen que tratar a diario con individuos de entornos culturales diversos (Van der Zee y Van Oudenhoven, 2013). Esto incluye a cualquier persona que participe o lidere un equipo multicultural, que viaje con frecuencia al extranjero por trabajo o que trate de forma habitual con personas de distintas culturas, ya sea dentro o fuera de las propias fronteras. Es importante destacar que hoy en día prácticamente cualquier trabajador entra, en mayor o menor grado, en una de estas categorías. Según McAllister, Whiteford, Hill, Thomas y Fitzgerald (2006), pocos profesionales trabajan ya en entornos monoculturales. A pesar de ello, muchas empresas siguen funcionando bajo el supuesto de que solo los empleados móviles, aquellos que se van a trasladar a otros países, requieren de atención especial en este sentido. Por esta razón, solo algunas compañías tratan de seleccionar empleados para estas misiones atendiendo a su mentalidad global, siendo los únicos que reciben algún tipo de formación intercultural. Para Peltokorpi y Froese (2012), algunas organizaciones incluso consideran que la voluntad del empleado para expatriarse es suficiente garantía de éxito y, por lo tanto, no consideran necesario identificar este tipo de talento ni ofrecer formación.

Sin embargo, dada la heterogeneidad multicultural en muchas regiones del mundo y el rápido crecimiento de la globalización, Leung et alii (2014) señalan que la competencia intercultural no se debe restringir únicamente a los expatriados. De hecho, debería extenderse a cualquier persona que estudie o trabaje en un entorno multicultural, ya sea dentro o fuera de sus fronteras.

El estudio del British Council y las consultoras Booz Allen Hamilton e Ipsos Public Affairs, Culture at Work, presenta de manera detallada la importancia de la competencia intercultural en los entornos laborales. Se basa en una encuesta realizada en a 367 gerentes de recursos humanos de grandes empresas empleadoras en nueve países diferentes: Brasil, China, India, Indonesia, Jordania, Sudáfrica, Emiratos Árabes Unidos, el Reino Unido y Estados Unidos (British Council et alii, 2013). A través de esta investigación, los gerentes definieron a un individuo con habilidades interculturales como alguien que a) es consciente de su propia cultura; b) entiende y acepta las diferencias culturales; c) se adapta a diferentes 
culturas; d) es respetuoso y flexible; e) habla idiomas; f) adapta su estilo de comunicación al contexto en el que se encuentra; g) genera confianza; h) escucha y observa, i) trabaja bien en equipos diversos; j) tolera la ambigüedad; k) está abierto a nuevas ideas y l) se interesa por continuar aprendiendo. Los encuestados parecían coincidir en que estas competencias son vitales no solo para permitir transacciones de negocios internacionales, sino para desarrollar relaciones a largo plazo con clientes y proveedores, mejorar el trabajo en equipo y la comunicación, promover la creatividad y reducir el conflicto. El estudio puso de relieve los beneficios específicos que la competencia intercultural de los empleados supone para una organización. Entre ellos mencionaron los siguientes: mayor eficacia de los equipos; mejor reputación de la empresa; nuevos clientes y mantenimiento de la confianza de los antiguos; buena comunicación con los socios; satisfacción entre los empleados y mayor permanencia de estos en la empresa; mayor productividad, y aumento de las ventas.

Por el contrario, reconocían que la falta de competencias interculturales aumentaba los problemas en la comunicación; provocaba más conflictos; perjudicaba la reputación de la empresa; suponía pérdida de clientes, insensibilidad con los socios y errores en los proyectos. El estudio, además, señala que una de las principales preocupaciones de estas empresas es encontrar a los candidatos apropiados para competir en el mundo global. Sin embargo, curiosamente, a la hora de seleccionar candidatos más de la mitad de los encuestados reconocían que sus procesos de selección de personal no detectaban suficientemente bien estas habilidades interculturales. Solo los empleadores en Jordania, la India y los Emiratos Árabes Unidos estaban satisfechos con sus procesos de selección a este respecto. En general, y a pesar de reconocer la importancia de estas competencias, solo un tercio de los encuestados afirmó que buscaban este tipo de habilidades de manera activa (British Council et alii, 2013).

En cualquier caso, esta relación entre la competencia intercultural y otras competencias profesionales interpersonales más tradicionales se encuentra también en otros autores como Crossman y Clarke (2010) o Jones (2011; 2013), que señalan que las competencias que se desarrollan en un entorno internacional vienen acompañadas de mejoras en las habilidades comunicativas y en la capacidad de negociación internacional. Asimismo, para Maddux, Adam y Galinsky (2010), los entornos culturales diversos estimulan también el desarrollo de formas más creativas de pensar, y para Page (2007), los equipos culturalmente diversos resuelven problemas con más eficacia que los homogéneos. 


\section{Modelos de competencia intercultural}

A continuación, analizaremos algunos de los modelos de competencia intercultural más relevantes que, como explica Deardorff (2006), son un intento por parte de los diferentes estudiosos de organizar los componentes de la competencia intercultural. Leung et alii (2014), con el fin de trabajar los conceptos y modelos de competencia intercultural de una manera práctica, destacan la necesidad de clasificarlos en tres grandes grupos o dominios, ya que, según ellos, existen más de treinta modelos de competencia intercultural y más de trescientos constructos presentes en la literatura para definir estas características o habilidades interculturales. El primer grupo está formado por características o rasgos interculturales, el segundo está basado en actitudes y una visión del mundo intercultural, y el tercero se centra en capacidades o habilidades interculturales.

Según su clasificación, el primer grupo está representado, entre otros, por Van der Zee y Van Oudenhoven (2000), a través de su modelo de personalidad multicultural basado en rasgos de personalidad relativamente estables y significativamente relacionados con la adaptación sociocultural, las aspiraciones internacionales, la satisfacción en el trabajo en entornos internacionales, la actividad multicultural y el éxito en equipos multiculturales, como hemos mencionado anteriormente. En el segundo dominio encontramos los modelos relacionados con una visión del mundo intercultural, es decir, una actitud positiva hacia contactos e interacciones interculturales. Este grupo está representado por Bennett (1986; 1993) y su modelo de desarrollo de la sensibilidad intercultural. Bennett entiende la competencia intercultural como un continuo desarrollado a lo largo de una serie de fases que conduce al individuo desde el etnocentrismo al etnorrelativismo, convirtiendo su visión del mundo en más sofisticada y compleja según avanza por estas fases (Hammer et alii, 2003). El tercer grupo se basa en el modelo de inteligencia cultural de Earley y Ang (2003), en el que encontramos las capacidades que permiten a un individuo funcionar de manera efectiva en un entorno cultural diverso.

Asimismo, existen también varios modelos mixtos, como, por ejemplo, el que Gertsen (1990), pionero en su campo, desarrolla dada la importancia creciente de la selección y entrenamiento de trabajadores expatriados. A partir de su modelo, han sido desarrollados muchos otros modelos similares. Este modelo parte de la base de que la competencia intercultural puede ser aprendida y enseñada, y centra su atención en tres componentes básicos analizados recurrentemente en la literatura: afectivo, cognitivo y conativo o comunicativo conductual. El componente afectivo es el que hace referencia a la personalidad y está relacionado con la sensibilidad, la motivación, el respeto y la comprensión hacia otras culturas, el 
interés por el contacto cultural, una actitud positiva hacia la cultura extranjera, así como la inclinación a aceptar diferencias culturales y a respetar otras costumbres. El componente cognitivo está relacionado con conocimiento y la noción de la cultura, tanto la propia como la ajena. Se refiere tanto a la manera en la que los individuos adquirimos la información y la agrupamos en categorías como al conocimiento general adquirido sobre las diferencias culturales o estilos de comunicación. También hace referencia al conocimiento de las distintas culturas, sus formas de organización, sus valores, normas y comportamientos, así como a la interacción entre ellas. El componente comunicativo conductual está relacionado con la competencia o habilidad para poner en práctica la comunicación apropiada, tanto verbal como no verbal, en los distintos contextos interculturales. Este componente es fundamental para poder expresar las habilidades afectivas (empatía, tolerancia o respeto por otros) y cognitivas, y, por tanto, ser un comunicador intercultural eficiente. Requiere estrategias para evitar y resolver malentendidos y para construir y mantener relaciones sociales. Según Gertsen, por tanto, la competencia intercultural es el resultado de la interacción de estas tres dimensiones.

También Bird et alii (2010) desarrollan un modelo mixto basado en competencias de liderazgo global que unifica tres dominios (características, visiones del mundo y capacidades) sintetizando la teoría y los estudios relacionados con el liderazgo y expatriación. La tabla 1 resume algunos ejemplos de los modelos más relevantes de competencia intercultural.

Tabla 1. Modelos de competencia intercultural

\begin{tabular}{|l|l|l|}
\hline \multicolumn{1}{|c|}{ Modelo } & \multicolumn{1}{|c|}{ Autores } & \multicolumn{1}{c|}{ Características } \\
\hline $\begin{array}{l}\text { Modelo de } \\
\text { competencia } \\
\text { intercultural }\end{array}$ & Gertsen $(1990)$ & $\begin{array}{l}\text { Analiza tres componentes básicos: afec- } \\
\text { tivo, cognitivo y conativo o comunicativo } \\
\text { conductual }\end{array}$ \\
\hline $\begin{array}{l}\text { Desarrollo de } \\
\text { la sensibilidad } \\
\text { intercultural }\end{array}$ & $\begin{array}{l}\text { Hammer y Bennett } \\
(1986 ; 1998)\end{array}$ & Actitudes o visión del mundo intercultural \\
\hline $\begin{array}{l}\text { Personalidad } \\
\text { multicultural }\end{array}$ & $\begin{array}{l}\text { Van der Zee y } \\
\text { Van Oudenhoven } \\
(2000)\end{array}$ & Características o rasgos interculturales \\
\hline $\begin{array}{l}\text { Inteligencia } \\
\text { cultural }\end{array}$ & Earley y Ang (2003) & Capacidades o habilidades interculturales \\
\hline $\begin{array}{l}\text { Competencias } \\
\text { de liderazgo } \\
\text { global }\end{array}$ & $\begin{array}{l}\text { Bird, Mendenhall, } \\
\text { Stevens y Oddou } \\
(2010)\end{array}$ & $\begin{array}{l}\text { Sintetiza teoría e investigación sobre lide- } \\
\text { razgo global y expatriación y unifica caracte- } \\
\text { rísticas, visiones del mundo y capacidades }\end{array}$ \\
\hline
\end{tabular}




\section{Herramientas para identificar y evaluar la competencia intercultural}

A continuación abordaremos la identificación y evaluación de la competencia intercultural a partir de distintas aproximaciones. Se trata de dar respuesta a una cuestión fundamental: ¿qué sistemas de medida son útiles y fiables para medir el rendimiento de un individuo en un entorno intercultural, ya sea dentro o fuera de su país?

Cada una de las grandes tres categorías de modelos detalladas por Leung et alii (2014) plasma su marco conceptual en unas formas específicas para medir la competencia intercultural. Van der Zee y Van Oudenhoven elaboran el Cuestionario de Personalidad Multicultural (MPQ) (2000), Hammer y Bennett desarrollan el Inventario de Desarrollo Intercultural (IDI) (1998), y Ang et alii (2007), la Escala de Inteligencia Cultural (CQ). Para Leung et alii (2014), el CQ y el MPQ son los modelos más prometedores y acertados de la competencia intercultural.

El MPQ tiene como objetivo medir, en palabras de los propios autores, la efectividad multicultural, y contribuir a la selección de empleados internacionales, además de ser utilizado como herramienta para diagnosticar y evaluar la necesidad del entrenamiento individual (Van der Zee y Van Oudenhoven, 2000). Con respecto a la validación y fiabilidad del cuestionario, varias investigaciones utilizan como muestra a estudiantes de distintas culturas y se enfocan también en la selección de personal y en los expatriados: Van der Zee et alii (2003), Van Oudenhoven et alii (2003), Leone et alii (2005) y Leong (2007) y Van Oudenhoven, Timmerman y Van der Zee (2007), entre otros muchos. Van der Zee, Atsma y Brodbeck (2004) analizan la identidad social y la personalidad de estudiantes de negocios que trabajaban juntos en equipos de trabajo culturalmente diversos. Van der Zee, Van Oudenhoven y De Grijs (2004) examinan las diferencias individuales a través de la evaluación de las reacciones emocionales en situaciones interculturales. En este caso, proporcionan a los estudiantes una descripción de una situación potencialmente poco o muy estresante y evalúan su reacción. Los estudiantes que puntúan alto en el MPQ reaccionan de manera más positiva ante situaciones interculturales estresantes. Van der Zee y Van de Gang (2007) también estudian las reacciones emocionales en entornos culturalmente diversos.

Milton Bennett (1986), a su vez, contribuye al estudio de la sensibilidad y la competencia intercultural con su modelo de desarrollo de la sensibilidad intercultural (Developmental Model of Intercultural Sensitivity, DMIS). El DMIS se crea para dar una explicación a cómo la gente interpreta las diferencias culturales. Bennett (1993), en sus investigaciones sobre el etnocentrismo y etnorrelativismo, 
expone su modelo y distingue seis fases en el desarrollo de la sensibilidad intercultural: negación, defensa, minimización, aceptación, adaptación e integración. Un individuo va pasando por estas fases a medida que experimenta y comprende mejor las diferencias culturales. Este marco teórico proporciona las bases para un sistema de medida que evalúa esa disposición hacia las diferencias culturales. El cuestionario desarrollado a partir de esta teoría se conoce como el Inventario de Desarrollo Intercultural (Intercultural Development Inventory, IDI) (Hammer et alii, 2003).

Un concepto introducido por los investigadores Earley y Ang (2003) es el de la inteligencia cultural o cociente cultural (Cultural Intelligence, CQ). Ward, Fischer, Lam y Hall (2009), a través de varios estudios, tratan de evaluar la validez del modelo diseñado en 2004 por Ang, Van Dyne, Koh y Ng para medir el CQ. La inteligencia cultural se define como la capacidad de una persona para adaptarse de una manera eficaz a contextos culturales diferentes. Representa un concepto multidimensional y mide cuatro componentes esenciales: cognitivo, metacognitivo, motivacional y conductual. El cognitivo se refiere al conocimiento que un individuo tiene de las normas, prácticas o convenciones en un contexto nuevo. El metacognitivo es la conciencia cultural que un individuo tiene cuando interactúa con gente de otras culturas. El componente motivacional se define como la intención o interés de una persona en conocer y funcionar de manera efectiva en otra cultura. Finalmente, el relativo a la conducta se entiende como la flexibilidad a la hora de demostrar comportamientos apropiados en otras culturas (Ward et alii, 2009).

También existen otros sistemas de medida como el Inventario de Sensibilidad Intercultural (Intercultural Sensitivity Inventory, ICSI), la Escala Intercultural de Potencial para la Adaptación (Intercultural Adjustment Potential Scale, ICAPS), la Escala de Sensibilidad Intercultural (Intercultural Sensitivity Scale, ISS) y el método basado en incidentes críticos. Así pues, autores como Bhawuk y Brislin (1992) destacan que el concepto de sensibilidad intercultural no es fácil de medir, entre otras cosas, porque no está claro a qué se tiene que ser sensible exactamente. En su trabajo proponen una herramienta conocida como el Inventario de Sensibilidad Intercultural (ICSI), que analiza las siguientes cuestiones. En primer lugar, la capacidad de la gente de entender las distintas maneras de hacer las cosas dependiendo de si se encuentran, por ejemplo, en una sociedad colectivista o individualista. En segundo lugar, la apertura de mente hacia las diferencias culturales, $y$, por último, la flexibilidad con respecto a comportamientos desconocidos o poco familiares.

Matsumoto et alii (2001), en su estudio, presentan una herramienta diseñada para medir el potencial de un individuo a la hora de adaptarse a un nuevo entor- 
no intercultural. Describen la adaptación intercultural alegando que esta va más allá del conocimiento específico de una cultura. La clave para la adaptación es la identificación de ciertas variables psicológicas que ellos realizan a través de un análisis de encuentros interculturales. Según los autores, debido a las diferencias de idioma, comportamientos no verbales, valores, actitudes, reglas, sistemas y otras manifestaciones de la cultura, la adaptación intercultural está repleta de conflictos, frustración y lucha. Para ello diseñan una herramienta conocida como la Escala Intercultural de Potencial para la Adaptación (ICAPS) y en su artículo describen el desarrollo y validación de esta escala a través de ocho estudios que miden el potencial de la adaptación intercultural de residentes temporales e inmigrantes japoneses a Estados Unidos. Para los autores, la adaptación intercultural es de crucial importancia no solo para aquellos que se están adaptando a nuevas y diferentes culturas, sino también para profesores, administradores, personas de negocios e individuos que a diario viven, trabajan y se divierten con personas de diferentes culturas (Matsumoto et alii, 2001). En 2003 presentan seis estudios adicionales que proporcionan una prueba más de la fiabilidad y validez del ICAPS con la particularidad de que sus resultados muestran cómo el cuestionario, originalmente desarrollado para su uso con japoneses, es válido también para otras culturas (Matsumoto et alii, 2003).

La Escala de Sensibilidad Intercultural de Chen y Starosta (2000) tiene por objetivo definir el nivel de sensibilidad intercultural de los participantes y la capacidad de discriminar y experimentar las diferencias culturales relevantes.

Sobre la eficacia intercultural también hablan Herfst, Van Oudenhoven y Timmerman (2008), aportando instrumentos de entrenamiento intercultural basado en incidentes críticos. El análisis de los incidentes críticos es una metodología de trabajo utilizada para observar y reflexionar sobre el comportamiento humano. Estas observaciones ayudan a resolver y visibilizar problemas prácticos, así como desarrollar principios básicos. Una de las técnicas utilizadas es pedir a los encuestados que describan una experiencia personal especialmente útil en el campo de la comunicación y gestión intercultural.

Finalmente, Matsumoto y Hwang (2013), en un estudio sobre la evaluación de la competencia intercultural, comparan diez modelos diferentes, coincidiendo así con Leung et alii (2014) en que tanto el CQ como el MPQ (además del ICAPS) son los modelos y herramientas que han presentado más pruebas de éxito en la medición de la competencia intercultural. La tabla 2 muestra el resumen de las principales herramientas para medir la competencia intercultural.

Tabla 2. Herramientas para identificar y evaluar la competencia intercultural

\begin{tabular}{l|l|l} 
Herramienta & Autores & Características
\end{tabular}




\begin{tabular}{|c|c|c|}
\hline $\begin{array}{l}\text { Inventario de Sensi- } \\
\text { bilidad Intercultural } \\
\text { (ICSI) }\end{array}$ & $\begin{array}{l}\text { Bhawuk y } \\
\text { Brislin (1992) }\end{array}$ & $\begin{array}{l}\text { Mide la capacidad de entender las distintas } \\
\text { maneras de comportarse, la apertura de men- } \\
\text { te hacia las diferencias culturales y la flexibili- } \\
\text { dad con respecto a comportamientos descon- } \\
\text { ocidos o poco familiares }\end{array}$ \\
\hline $\begin{array}{l}\text { Inventario de Desar- } \\
\text { rollo Intercultural } \\
\text { (IDI) }\end{array}$ & $\begin{array}{l}\text { Hammer } \\
\text { y Bennett } \\
(1998)\end{array}$ & $\begin{array}{l}\text { Distingue seis fases en el desarrollo de la } \\
\text { sensibilidad intercultural: negación, defensa, } \\
\text { minimización, aceptación, adaptación e inte- } \\
\text { gración }\end{array}$ \\
\hline $\begin{array}{l}\text { Escala de Sensibi- } \\
\text { lidad Intercultural } \\
\text { (ISS) }\end{array}$ & $\begin{array}{l}\text { Chen y Staros- } \\
\text { ta }(2000)\end{array}$ & $\begin{array}{l}\text { Define el nivel de sensibilidad intercultural } \\
\text { de y la capacidad de discriminar y experimen- } \\
\text { tar las diferencias culturales relevantes }\end{array}$ \\
\hline $\begin{array}{l}\text { Cuestionario de } \\
\text { Personalidad Multi- } \\
\text { cultural (MPQ) }\end{array}$ & $\begin{array}{l}\text { Van der Zee y } \\
\text { Van Ouden- } \\
\text { hoven (2000; } \\
\text { 2001) }\end{array}$ & $\begin{array}{l}\text { Mide cinco características o rasgos de la per- } \\
\text { sonalidad multicultural: empatía cultural, } \\
\text { apertura mental, iniciativa social, estabilidad } \\
\text { emocional y flexibilidad }\end{array}$ \\
\hline $\begin{array}{l}\text { Escala Intercultural } \\
\text { de Potencial para } \\
\text { la Adaptación, } \\
\text { (ICAPS) }\end{array}$ & $\begin{array}{l}\text { Matsumoto, } \\
\text { LeRoux, Rat- } \\
\text { zlaff, Tatani, } \\
\text { Uchida, Kim y } \\
\text { Araki (2001) }\end{array}$ & $\begin{array}{l}\text { Identifica ciertas variables psicológicas a } \\
\text { través de un análisis de encuentros intercul- } \\
\text { turales }\end{array}$ \\
\hline $\begin{array}{l}\text { Escala de Inteligen- } \\
\text { cia Cultural (CQ) }\end{array}$ & $\begin{array}{l}\text { Ang et alii } \\
(2007)\end{array}$ & $\begin{array}{l}\text { Representa un concepto multidimensional y } \\
\text { mide cuatro componentes esenciales: cogniti- } \\
\text { vo, metacognitivo, motivacional y conductual }\end{array}$ \\
\hline $\begin{array}{l}\text { Instrumentos basa- } \\
\text { dos en incidentes } \\
\text { críticos }\end{array}$ & $\begin{array}{l}\text { Herfst, Van } \\
\text { Oudenhoven } \\
\text { y Timmerman } \\
(2008)\end{array}$ & $\begin{array}{l}\text { Metodología utilizada para observar y } \\
\text { reflexionar sobre experiencias personales y } \\
\text { problemas prácticos }\end{array}$ \\
\hline
\end{tabular}

\section{Conclusiones}

La literatura nos presenta la necesidad de desarrollar la competencia intercultural en ámbitos profesionales globales y multiculturales. La mayor parte de los autores combinan las competencias profesionales tradicionales con el más reciente y novedoso componente cultural. Parece claro que este tipo de competencia engloba ya, o englobará en el futuro muy cercano, muchas de las habilidades profesionales más demandadas en la actualidad (creatividad, trabajo en equipo, liderazgo, comunicación, etc.). Dicho de otro modo, es muy posible que en las próximas décadas la comunicación efectiva sea casi siempre intercultural, el trabajo sea mayoritariamente en equipos culturalmente diversos y la empatía, entre otras, se considere fundamental para adaptarse y trabajar en los entornos heterogéneos culturalmente. Así pues, las competencias profesionales necesarias en la actuali- 
dad apuntan a convertirse en una combinación entre las competencias profesionales tradicionales y la competencia intercultural. El componente intercultural se manifiesta, por tanto, en la mayor parte de los entornos profesionales globales.

Sin embargo, con respecto a la identificación y evaluación de la competencia intercultural en ámbitos laborales, debemos destacar varios problemas importantes. En primer lugar, parece existir una falta de concienciación a nivel empresarial sobre la importancia de la competencia intercultural en entornos de trabajo cada día más multiculturales. Esto, lamentablemente, impide el avance de una disciplina esencial para el funcionamiento efectivo de las organizaciones en el mundo. Una de las razones principales por las que este fenómeno tiene lugar es, entre otras cosas, porque existe una tendencia a la minimización de las diferencias culturales. En algunos casos, esto ocurre por una falsa sensación de homogeneización cultural provocada por la percepción que se tiene del mundo global. Creemos, como explica Bennett (1993), que los seres humanos somos esencialmente iguales y que las diferencias culturales son más bien superficiales y no tan relevantes. En otros casos, se minimizan las diferencias, ya que se teme ofender a otras personas al no saber abordar la cuestión cultural.

En segundo lugar, ser consciente de la necesidad de identificar y desarrollar la competencia intercultural es complicado cuando, como hemos visto, los conceptos de cultura y competencia intercultural no están claramente definidos. De ahí que la evaluación de este tipo de competencia no sea tarea fácil. El problema se agudiza, además, ya que los estudios de investigación con profesionales son limitados.

Finalmente, a esto se suma que en muchas organizaciones la selección del talento o entrenamiento intercultural ni siquiera es prioritario, una vez más, porque no se sabe exactamente de qué se trata, ni cuáles son sus beneficios. Los malentendidos culturales y los problemas de comunicación intercultural que acaban a diario con importantes negocios son muy sutiles y difíciles de reconocer. La mayor parte de las veces estos fracasos en las negociaciones se atribuyen a cuestiones económicas o políticas, dejando a un lado la influencia del factor cultural y la (falta) de competencias profesionales interculturales.

Así pues, el intento por clasificar los componentes culturales a través de los distintos modelos de competencia intercultural es muy útil, ya que permite clasificar y comprender mejor este tipo de habilidades. Asimismo, facilitan el desarrollo de herramientas que, si bien se encuentran todavía en fases iniciales de implementación, ayudan a identificar y evaluar la competencia intercultural en organizaciones internacionales. Por tanto, si bien es cierto que queda mucho camino por recorrer, estas herramientas y los modelos que las sustentan son un 
aporte fundamental al estudio de esta reciente disciplina. En cualquier caso, se necesitan muchos más estudios empíricos con profesionales de todo tipo para llegar a conclusiones sólidas y desarrollar herramientas más sofisticadas y fiables.

En conclusión, identificar, fomentar y entrenar las habilidades interculturales en los futuros profesionales, con el fin de disponer de talento global y un buen rendimiento profesional en nuestras organizaciones y empresas el día de mañana, debe convertirse en una prioridad para todas las organizaciones en la actualidad. Una vez más las organizaciones y la academia deben buscar soluciones conjuntas a los retos que presenta el mundo globalizado. 


\section{Bibliografía}

Akri, M. (2013)."Study Abroad and Cultural Learning Through Fulbright and Other International Scholarships: A holistic student development". Journal of International Students, 3 (1), 1-9.

Ang, S.; Van Dyne, L.; Кoh, C.; Ng, K. Y.; Templer, K. J.; Tay, C. Y Chandrasekar, N. A. (2007)."Cultural Intelligence: Its measurement and effects on cultural judgment and decision making, cultural adaptation and task performance". Management and Organization Review, 3 (3), 335-371.

Arthur, W. y Bennett, W. (1995). "The International Assignee: The relative importance of factors perceived to contribute to success". Personnel Psychology, 48 (1), 99-114.

Bakalis, S. y Joiner, T. A. (2004). "Participation in Tertiary Study Abroad Programs: The role of personality." International Journal of Educational Management, 18 (5), 286-291.

Barrett, M.; Byram, M.; Lázár, I.; Mompoint-Gaillard, P. y Philippou, S. (2013). Developing Intercultural Competence through Education. Strasbourg: Council of Europe.

Bhawuk, D. y Brislin, R. (2000). “Cross-Cultural Training: A review.” Applied Psychology, 49 (1), 162-191.

Behrnd, V. y Porzelt, S. (2012). "Intercultural Competence and Training Outcomes of Students With Experiences Abroad”. International Journal of Intercultural Relations, 36, 213-223.

Bennett, M.J. (1986).“A Developmental Approach to Training for Intercultural Sensitivity". International Journal of Intercultural Relations, 10 (2), 179-196.

Bennett, M. J. (1993). “Towards Ethnorelativism: A developmental model of inter- cultural sensitivity." En R. M. PAIGE (ed.). Education for the Intercultural Experi-ence (2. ed., pp. 21-71). Yarmouth, ME: Intercultural Press.

Bennett, M. J. (1998) "Overcoming the Golden Rule: Sympathy and empathy". En M. J. Bennett (ed.). Basic Concepts of Intercultural Communication: Selected Readings, 5 (pp. 191-214). Yarmouth, ME: Intercultural Press.

Bird, A.; Mendenhall, M.; Stevens, M. J. y Oddou, G. (2010). “Defining the content domain of intercultural competence for global leaders". Journal of Managerial Psychology, 25 (8), 810-828.

British Council (2013). Culture at Work: The value of intercultural skills in the workplace. Recuperado de <https://www.britishcouncil.org/sites/default/ files/-culture-at-work-report-v2.pdf $>$.

Bücker, J. y Poutsma, E. (2010). "Global Management Competencies: A theoretical foundation". Journal of Managerial Psychology, 25 (8), 829-844. 
Busch, D. (2009). "What Kind of Intercultural Competence will Contribute to Students' Future Job Employability?” Intercultural Education, 20 (5), 429-438.

Chiu, Chi-Yue; Lonner, W. J; Matsumoto, D. y Ward, C. (2013). "CrossCultural Competence: Theory, research, and application". Journal of CrossCultural Psychology, 44 (6), 843-848.

Crossman, J. E. y Clarke, M. (2010). "International Experience and Graduate Employability: Stakeholder perceptions on the connection". Higher Education, 59 (5), 599-613.

Deardorff, D. K. (2006). "Identification and Assessment of Intercultural Competence as a Student Outcome of Internationalization". Journal of Studies in International Education, 10 (3), 241-266.

Dirección General de Industria y de la Pequeña y Mediana Empresa (2018). Retrato de las PYME. Madrid: Ministerio de Industria, Turismo y Comercio.

EARley, P. C. y Ang, S. (2003). Cultural Intelligence: Individual interactions across cultures. Stanford, CA: Stanford University Press.

Espinosa Romero, M. (2009). Los procesos de internacionalización empresarial y la gestión de personas. Madrid: Fundación EOI.

FAnjul, E. (2010). "Factores culturales e internacionalización de la empresa”. Información comercial española, (856), 7-20.

Gertsen, M. C. (1990). "Intercultural Competence and Expatriates". The International Journal of Human Resource Management, 1 (3), 341-362.

Hammer, M. R. y Bennett, M. J. (1998). The Intercultural Development Inventory (IDI) Manual. Portland, OR: The Intercultural Communication Institute.

Hannigan, T. P. (1990). "Traits, attitudes, and skills that are related to intercultural effectiveness and their implications for cross-cultural training: A review of the literature". International Journal of Intercultural Relations, 14 (1), 89-111.

Hawes, F. y Kealey, D. J. (1981). “An Empirical Study of Canadian Technical Assistance: Adaptation and effectiveness on overseas assignment". International Journal of Intercultural Relations, 5 (3), 239-258.

Hall, E. T. (1982). The Hidden Dimension. Garden City, N.Y: Anchor Books Editions.

Hammer,M.R.; Bennett, M.J.y Wiseman, R.(2003)."Measuring Intercultural Sensitivity: The intercultural development inventory". International Journal of Intercultural Relations, 27 (4), 421-443. 
Herfat, S. L*; Van Oudenhoven, J. P. y Timmerman, M. E. (2008). "Intercultural Effectiveness Training in three Western immigrant countries: A cross-cultural evaluation of critical incidents". International Journal of Intercultural Relations, 32 (1), 67-80.

Hofstede, G. (2001). Culture's Consequences: Comparing values, behaviors, institutions and organizations across nations. Thousand Oaks, CA: Sage Publications, Inc.

Hofstede, G*; Hofstede, G. J. y Minkov, M. (2010). Cultures and Organizations: Software of the Mind ( $3+^{\mathrm{a}}$ ed.). London: McGraw-Hill.

Irving, J. A. (2010). "Educating Global Leaders: Exploring intercultural competence in leadership education". Journal of International Business and Cultural Studies, 3, 1-14.

Johnson, J.P.; Lenartowicz, T.y Apud,S.(2006)."Cross-Cultural Competence in International Business: Toward a definition and a model". Journal of International Business Studies, 37 (4), 525-543.

Jones, E. (2011). "Internationalisation, Multiculturalism: A global outlook and employability”. Assessment, Teaching E Learning Journal, 11, 21-49.

Jones, E. (2013). "Internationalization and Employability: The role of intercultural experiences in the development of transferable skills". Public Money E Management, 33 (2), 95-104.

Kealey, D. J. y Ruben, B. D. (1983). "Cross-Cultural Personnel Selection Criteria, Issues And Method”. En D. Landis y R. W. Brislin (eds.). Handbook of intercultural training: Issues in theory and design, 1 (pp. 155-175). New York: Pergamon Press.

Leung, K.; Ang, S. y TAn, M. L. (2014). "Intercultural competence”. The Annual Review of Organizational Psychology and Organizational Behavior, 1 (1), 489519.

Leone, L.; Van der Zee, K. I.; Van Oudenhoven, J. P.; Perugini, M. Y Ercolani, A. P. (2005). "The Cross-Cultural Generalizability and Validity of the MPQ". Personality and Individual Differences, 38 (6), 1449-1462.

Leong, C. H. (2007). "Predictive Validity of the Multicultural Personality Questionnaire: A longitudinal study on the socio-psychological adaptation of Asian undergraduates who took part in a study-abroad program". International Journal of Intercultural Relations, 31 (5), 545-559.

Liv, S* Volcic, Z*y Gallois, C.(2015). Introducing Intercultural Communication: Global cultures and contexts. Thousand Oaks, CA: Sage.

Llamazares García-Lomas, O. (2011). "La influencia de la cultura en los negocios internacionales". Boletín económico de ICE, Información Comercial Española, 47-52. 
McAllister, L.; Whiteford, G.; Hill, B;; Thomas, N. y Fitzgerald, M. (2006). "Reflection in Intercultural Learning: examining the international experience through a critical incident approach". Reflective Practice, 7 (3), 367 381.

Maddux, W. W.; Adam, H. y Galinsky, A. D. (2010). "When in Rome... Learn Why the Romans Do What They Do: How multicultural learning experiences facilitate creativity". Personality and Social Psychology Bulletin, 36 (6),731-741.

Matsumoto, D.; LeRoux, J. A.; Imamoto, M.; Choi, J. W*; Rogers, D;; Tatani, H. y Uchida, H. (2003). "The Robustness of the Intercultural Adjustment Potential Scale (ICAPS): The search for a universal psychological engine of adjustment." International Journal of Intercultural Relations, 27 (5), 543-562.

Matsumoto, D.; LeRoux, J*; Ratzlaff, C.; Tatani, H.; Uchida, H.; Kim, C. y Araki, S. (2001). "Development and Validation of a Measure of Intercultural Adjustment Potential in Japanese Sojourners: The Intercultural Adjustment Potential Scale (ICAPS)". International Journal of Intercultural Relations, 25 (5), 483-510.

Matsumoto,D.y Hwang, H.C.(2013)."Assessing Cross-CulturalCompetence: A review of available tests". Journal of Cross-Cultural Psychology, 44 (6), 849873.

Morrison, T.; Conaway, W. A. y Borden, G. A. (2006). Kiss, bow, or shake hands. Avon, MA: Adams Media.

Page, S. E. (2007). The Difference: How the Power of Diversity Creates Better Groups, Firms, Schools, and Societies. Princeton, NJ: Princeton University Press.

Peltokorpi, V+y Froese, F+ J. (2012). “The Impact of Expatriate Personality Traits on Cross-Cultural Adjustment: A study with expatriates in Japan”. International Business Review, 21(4), 734-746.

Saso, C. E*; Errazu, D. V*; Berrozpe, T. I*, y Bahillo, C. G. (2017). "La competencia intercultural en las organizaciones: una aproximación teórica”. Revista Internacional de Organizaciones, (19), 75-92.

Shaffer, M. A.; Harrison, D. A.; Gregersen, H.; Black, J. S. y Ferzandi, L.A. (2006). "You Can Take It With You: Individual differences and expatriate effectiveness". Journal of Applied psychology, 91 (1), 109-125.

Tung, R. L. (1981). "Selection and Training of Personnel for Overseas Assignments". Columbia Journal of World Business, 16 (1), 68-78. 
Van der Zee, K. I. y Van Oudenhoven, J. P. (2000). "The Multicultural Personality Questionnaire: A multidimensional instrument of multicultural effectiveness". European Journal of Personality, 14 (4), 291-309.

Van der Zee, K. I. y Van Oudenhoven, J. P. (2001). “The Multicultural Personality Questionnaire: Reliability and validity of self- and other ratings of multicultural effectiveness". Journal of Research in Personality, 35, 278-288. VAn der Zee, K. I*; ZaAl, J. N. y Piekstra, J. (2003). "Validation of the Multicultural Personality Questionnaire in the Context of Personnel Selection”. European Journal of Personality, 17 (S1), S77-S100.

Van der Zee, K.y Van Oudenhoven,J.P.(2013)."Culture Shock or Challenge? The role of personality as a determinant of intercultural competence". Journal of Cross-Cultural Psychology, 44 (6), 928-940.

Van der Zee, K. I.; Van Oudenhoven, J. P.y De Grijs, E. (2004). "Personality, Threat, and Cognitive and Emotional Reactions to Stressful Intercultural Situations". Journal of Personality, 72 (5), 1069-1096.

Van der Zee, K.; Atsma, N. y Brodbeck, F+ (2004). “The Influence of Social Identity and Personality on Outcomes of Cultural Diversity in Teams". Journal of Cross-Cultural Psychology, 35 (3), 283-303.

Van der Zee, K. I. y Van der Gang, I. (2007). "Personality, Threat and Affective Responses to Cultural Diversity". European Journal of Personality, 21 (4), 453-470.

Van Oudenhoven, J. P.; Mol, S. y Van der Zee, K. I. (2003). "Study of the Adjustment of Western Expatriates in Taiwan Roc with the Multicultural Personality Questionnaire”. Asian Journal of Social Psychology, 6 (2), 159-170. Van Oudenhoven, J. P*; Timmerman, M. E. y Van der Zee, K. (2007). "Cross-Cultural Equivalence and Validity of the Multicultural Personality Questionnaire in an Interculltural Context". Journal of International Communication, 13 (2), 51-65.

Ward, C.; Fischer, R.; Zaid Lam, F. S. y Hall, L. (2009)."The Convergent, Discriminant, and Incremental Validity of Scores on a Self-Report Measure of Cultural Intelligence". Educational and Psychological Measurement, 69 (1), 85-105. 